\title{
ORIGINAL
}

\section{PADRÃO ALIMENTAR DE LACTENTES RESIDENTES EM ÁREAS PERIFÉRICAS DE FORTALEZA'}

\section{ALIMENTARY PROFILE OF INFANTS IN SLUM AREAS OF FORTALEZA, BRAZIL}

\author{
Nadia Tavares SOARES ${ }^{2}$ \\ Augusto Reinaldo Pimentel GUIMARÃES² \\ Helena Alves de Carvalho SAMPAIO² \\ Paulo César de ALMEIDA ${ }^{3}$ \\ Roberta Ribeiro $\mathrm{COELHO}^{4}$
}

RESUMO

\begin{abstract}
Este estudo caracterizou o padrão alimentar de 96 crianças menores de um ano assistidas pelo Fundo Cristão para Crianças nos bairros Álvaro Weyne e Presidente Kennedy, na cidade de Fortaleza, Brasil. Os dados foram levantados por meio de entrevista domiciliar, utilizando o método recordatório $24 \mathrm{~h}$ para conhecimento das quantidades dos alimentos consumidos, e um formulário contendo perguntas estruturadas sobre a prática do aleitamento materno e idade de introdução dos alimentos de desmame. Os resultados indicaram que o aleitamento materno misto predomina (68\%) sobre o aleitamento artificial (32\%) e exclusivo (10\%) e que 7\% das crianças nunca receberam leite materno. Porém, até o final do décimo mês de vida, 53\% das crianças ainda são amamentadas. Mingau lácteo, preparado com leite de vaca não modificado, constitui a alimentação básica de desmame. Do ponto de vista nutricional, as dietas são desbalanceadas, sendo o ferro o nutriente mais deficiente. Devem ser intensificados os esforços na promoção da alimentação infantil apropriada.
\end{abstract}

Termos de indexação: desmame, alimentos infantis, aleitamento materno, prevalência, lactente.

ABSTRACT

The objective of this study was to characterize the food practice of 96 infants attended by Children Christian Fund in Álvaro Weyne and President Kennedy Districts in Fortaleza, Brazil. The data were collected using a 24-hour diet recall method and a structured questionnaire about breast feeding and weaning practices. The results indicate that partial breast feeding is more frequent (68\%) than artificial feeding (32\%) and exclusive breast feeding (10\%) and $7 \%$ of the infants were never breast-fed. But, at the age of 10 months, 53\% of the infants are still being breast-fed. The porridge prepared with whole cow's milk is basically the entire weaning diet. From a nutritional point of view, the diets are unbalanced and iron is the most deficient nutrient. Intensive efforts for the promotion of adequate infant feeding practice are necessary.

Index terms: weaning, infant food, breast feeding, prevalence, infant.

\footnotetext{
${ }^{(1)}$ Condensação da dissertação de mestrado "Prática Alimentar de crianças menores de um ano; subsídio para implementação das ações em Nutrição e Saúde", apresentada e defendida na Universidade Estadual do Ceará (UECE) em março de 1997, reapresentada no Primeiro Seminário de Nutrição Materno-Infantil realizado em 10 de junho de 1997, no Instituto José Frota, sob a promoção do Departamento de Ciências da Nutrição da UECE e exposta na forma de poster no IV Congresso Brasileiro de Epidemiologia, realizado no Rio de Janeiro, período de 1 a 5 de agosto de 1998. Estudo financiado integralmente pela Fundação de Amparo à Pesquisa (FUNCAP) - processo 143/94 e conduzido junto a clientela vinculada ao Fundo Cristão para Crianças nos bairros Álvaro Weyne e Presidente Kennedy, Fortaleza, Ceará.

${ }^{(2)}$ Departamento de Nutrição, Universidade Estadual do Ceará. Rua Paranjana, 1700, Itaperi, 60740-400, Fortaleza, CE, Brasil. Correspondência para/Correspondence to: N.T.SOARES,

(3) Departamento de Saúde Pública, Universidade Estadual do Ceará.

${ }^{(4)}$ Bolsita do CNPq, Acadêmica do Curso de Nutrição, Universidade Estadual do Ceará.
} 


\section{INTRODUÇÃO}

As três maiores causas de mortalidade nos países em desenvolvimento são infecções respiratórias, diarréia e outras causas neonatais e perinatais. Juntas, representam $70 \%$ dos 12,9 milhões de mortes entre crianças menores de cinco anos. A Organização Mundial de Saúde estima que a desnutrição contribui com mais de $25 \%$ dessas mortes (Hufman \& Martin, 1994). Em nosso meio, o Instituto Brasileiro... (1992b) registrou que as infecções e a desnutrição foram conjuntamente responsáveis por $36 \%$ das mortes infantis ocorridas em 1986.

Embora haja referências de que esta problemática tenha raízes em uma rede complexa de fatores (grandes contigentes populacionais, inadequada infra-estrutura de saúde e educação, falta de vontade política dos governantes, limitados recursos agrícolas e socioeconômicos) (Chandra et al., 1988), a inadequada prática alimentar infantil aparece como uma das mais importantes razões para iniciar o círculo vicioso entre infecção e desnutrição (Kumar et al., 1992).

No entanto, a despeito desses conhecimentos, observa-se que poucos estudos científicos têm sido realizados no Brasil sobre a situação alimentar infantil (Shrimpton, 1986; Szarfarc et al., 1988; Prado et al., 1995). Os dados oficiais disponíveis, inclusive do Ceará (Instituto Brasileiro..., 1982, 1992a; Governo..., 1990a,b, 1995) retratam quase que exclusivamente a prática do aleitamento materno, deixando de lado outros elementos também importantes, como os aspectos qualitativos e quantitativos da alimentação artificial e o processo de desmame.

Os estudos sobre prática alimentar infantil, em especial os de prevalência, são essenciais antes da formulação de qualquer programa de intervenção nutricional e necessários para a adequada avaliação do impacto de qualquer medida implementada (Bavdekar et al., 1994; Coutsoudis et al., 1994). Portanto, a escassez desses dados repercute na tomada de decisões diárias de diversas instituições que atuam no tratamento e prevenção à desnutrição.

Diante do exposto, este estudo teve por objetivo caracterizar o padrão alimentar de crianças menores de um ano vinculadas ao Fundo Cristão para Crianças nos bairros Álvaro Weyne e Presidente Kennedy.

\section{CASUÍSTICA E MÉTODOS}

A casuística compreendeu todas as crianças menores de um ano de idade vinculadas a 10 unidades assistenciais do Fundo Cristão para Crianças (entidade não-governamental) localizadas em dois bairros periféricos (Álvaro Weyne e Presidente Kennedy) da cidade de Fortaleza, Ceará, Brasil. Ao todo, foram 110 crianças, sendo que 96 participaram efetivamente do estudo. Para o levantamento quantitativo dos dados alimentares, extraiu-se deste total uma amostra aleatória simples composta por $50 \%$ das crianças.

\section{Local}

Nos bairros Álvaro Weyne e Presidente Kennedy, o Fundo Cristão para Crianças mantém nove unidades assistenciais, denominadas de projetos e um Centro de Estimulação e Desenvolvimento Infantil (CEDI) voltados, principalmente, para a promoção das ações básicas de saúde e para atividades de reforço e estímulo à aprendizagem. As ações de saúde, tais como, educação e orientação em saúde e nutrição, monitoramento do crescimento e desenvolvimento; e acompanhamento da situação vacinal, são desenvolvidas sob o mesmo modelo da rede municipal de saúde. No entanto, há distinções na composição do quadro profissional e no envolvimento com a clientela, que no caso do Fundo Cristão para Crianças é mais próximo.

As sedes dos projetos situam-se em construções simples, de pequeno porte, similares às casas da comunidade. Suas equipes técnicas são compostas, basicamente por economistas domésticos, pedagogos e agentes de saúde. O médico figura no quadro, mas dando assistência semanal em algumas poucas Unidades.

O CEDI possui estrutura ampla, devidamente planejada para trabalhar o crescimento e o desenvolvimento da criança como um todo. Possui uma equipe multiprofissional (nutricionista, assistente social, pedagogo, fisioterapeuta, fonoaudiólogo, terapeuta ocupacional, psicólogo) e funciona como um semi-internato. O CEDI conta ainda com o trabalho de agentes de saúde e toda equipe auxiliar advinda da própria comunidade. Os projetos, diferentemente do CEDI, fazem acompanhamento pré-natal, e oferecem, de forma sistemática, cursos aos familiares das crianças (datilografia, alternativas alimentares, trabalhos manuais, farmácia viva).

Quase todas as crianças assistidas residem em casas de alvenaria (99\%), porém com precárias instalações sanitárias. A maioria (79\%) possui vaso sanitário sem descarga, eliminação dos dejetos feita em fossas rudimentares (não sépticas) (83\%), e limitações no abastecimento público de água e no destino do lixo. A famílias (79\%) apresentam até três salários mínimos como renda mensal, sendo freqüente rendimentos entre um e dois salários mínimos (40\%). Somente 14\% dos pais chegaram a concluir as oito séries do primeiro grau escolar (Soares, 1997).

Nesta localidade, o Fundo Cristão para Crianças assiste 3671 crianças de zero a 15 anos, sendo 1130 na faixa de zero a 5 anos, enquanto o censo de 1991 (Instituto Brasileiro..., 199-) aponta a existência de 5070 crianças de zero a 4 anos e 10381 de 5 a 14 anos. 
A captação da clientela é feita por moradores da comunidade, intitulados "representantes de rua", porém a inscrição das crianças se efetiva quando a renda per capita média da família é igual ou inferior à metade do salário mínimo vigente. O vínculo com o CEDI se processa por meio de encaminhamento feito pelos coordenadores dos projetos. Em caso da demanda ser maior que o número de vagas, leva-se em consideração a situação socioeconômica e o estado nutricional da criança, dando-se preferência aos mais necessitados. O CEDI comporta 504 crianças de zero a 6 anos, sendo 20 o número de vagas para bebês de zero a 1 ano.

\section{Coleta dos dados}

Os dados alimentares foram levantados em dois segmentos, por meio de entrevista domiciliar. O entrevistado-alvo foi o responsável direto pelo cuidado da criança, de preferência a mãe. No primeiro segmento, registrou-se em um formulário contendo perguntas estruturadas, informações relativas à prática do aleitamento materno e idade de introdução dos alimentos de desmame. No segundo segmento, obteve-se através do método recordatório de $24 \mathrm{~h}$, aplicado durante três dias não consecutivos, informações sobre o tipo, marca comercial e quantidade dos alimentos de desmame consumidos. Para garantir maior precisão no registro dos dados, foram utilizados medidores graduados, colheres e xícaras padrão, além de balanças eletrônicas portáteis (precisão 1 g). Foram utilizadas também ilustrações de porções e medidas de alimentos da publicação de Martins (1982).

\section{Análise dos dados}

A adequação dos dados de consumo alimentar foi estabelecida em comparação com as recomendações internacionais (National Research..., 1989) para energia, proteínas, cálcio, fósforo, ferro, vitamina C, tiamina, riboflavina, niacina e retinol. Para o cálculo de adequação da niacina levou-se em consideração o seu desdobramento a partir do aminoácido triptofano (Anderson et al., 1988)

O cálculo das adequações das dietas mistas (aleitamento materno parcial) foi feito através da ingestão estimada de leite materno. Para isto tomou-se por referência o número médio de mamadas encontrado entre as crianças em aleitamento parcial (5,3 vezes/dia); o conhecimento de que, com base em diversos estudos, o Conselho de Alimentação e Nutrição da Academia Nacional de Ciências dos EUA assume que crianças em aleitamento materno ingerem no primeiro e segundo semestre cerca de $750 \mathrm{ml}$ e $600 \mathrm{ml}$ de leite, respectivamente (Hervada \& Newman, 1992), e a referência de que em culturas onde a subnutrição materna é comum, as mulheres em amamentação exclusiva produzem $600 \mathrm{ml}$ de leite/dia, com sucção correspondente a 14 - 20 mamadas (Underwood \& Hofvander, 1982).
Considerando que a produção de leite referida por estes últimos autores não está tão distanciada da recomendação do Conselho Americano, que é mais recente e foi estabelecida com base em diversos estudos, optou-se em relacionar as 5,3 mamadas com 14 - 20 sucções para o consumo de $750 \mathrm{ml}$ no primeiro semestre e $600 \mathrm{ml}$ no segundo. O consumo médio de cada faixa etária foi analisado e somado às dietas de cada criança em aleitamento materno parcial (consumo de leite materno mais água ou qualquer outro alimento diferente do leite materno) (Brasil, 1994). O consumo médio de leite materno ficou estimado em $241 \mathrm{ml}$ para o primeiro semestre (199-284) e $193 \mathrm{ml}$ (159-227) para o segundo.

Os dados foram tabulados em microcomputador, utilizando-se o Programa de Apoio à Decisão em Nutrição (versão 2.5) para o cálculo da composição químico-nutricional e adequação das dietas. A composição do leite materno, contida neste programa, foi extraída do Manual do United States Departament of Agriculture. Fez-se análise descritiva (Programa Epi Info - versão 6.02) e inferencial (testes paramétricos e não paramétricos - Programa STATA).

\section{RESULTADOS E DISCUSSÃO}

\section{Prevalência do aleitamento materno}

A maioria das crianças (93\%) foi alimentada com leite materno no primeiro mês de vida, porém no período da coleta dos dados a prevalência encontrada foi $68 \%$. Em regime de aleitamento materno exclusivo, parcial e artificial registrou-se, respectivamente, $10 \%$, 58\% e $32 \%$ do total de crianças (Tabela 1). Entre as crianças na faixa de idade ideal para a prática do aleitamento materno exclusivo (60\%), encontrou-se $24 \%$ em aleitamento artificial e $60 \%$ em aleitamento parcial. Observa-se que o aleitamento materno parcial é predominante tanto no primeiro, como no segundo semestre de vida. Houve associação estatisticamente significante entre padrão alimentar e faixa etária $\left(\chi^{2}=9,015 ; p=0,01\right)$ (Tabela 1$)$.

Ressalta-se que entre as crianças em alimentação artificial $(n=32), 22 \%(n=7)$ nunca chegaram a receber leite materno. Mas, através da técnica da "tábua de vida", verificou-se que até o final do décimo mês, 53\% das crianças ainda são amamentadas, indicando que a duração mediana do aleitamento materno é superior a 10 meses. Este achado está acima da realidade brasileira, onde a mediana do aleitamento materno geral não ultrapassa 4,5 meses (Instituto Brasileiro..., 1992b).

O predomínio do aleitamento parcial em relação ao exclusivo, bem como a sua razoável duração, é sugestivo de que as mães valorizavam o leite materno, mas não a ponto de tê-lo como alimento único no período em que ele é indicado (zero a 6 meses). 
Tabela 1. Prática de aleitamento de crianças < 1 ano. Álvaro Weyne/Presidente Kennedy. Fortaleza, 1995.

\begin{tabular}{|c|c|c|c|c|c|c|}
\hline \multirow{3}{*}{ Prática de aleitamento } & \multicolumn{4}{|c|}{ Faixa etária } & \multirow{2}{*}{\multicolumn{2}{|c|}{ Total }} \\
\hline & \multicolumn{2}{|c|}{$\leq 6$} & \multicolumn{2}{|c|}{$>6$} & & \\
\hline & $\mathrm{n}$ & $\%$ & $\mathrm{n}$ & $\%$ & $\mathrm{n}$ & $\%$ \\
\hline Artificial & 14 & 24 & 17 & 45 & 31 & 32 \\
\hline Parcial & 35 & 60 & 21 & 55 & 56 & 58 \\
\hline Exclusivo & 9 & 16 & - & - & 9 & 10 \\
\hline Total & 58 & 100 & 38 & 100 & 96 & 100 \\
\hline
\end{tabular}

Nota: $\chi^{2}=9,015 ; p=0,01$

Além disso, pôde-se supor também, que as mães eram inseguras e/ou inadequadamente informadas quanto à prática do aleitamento materno exclusivo. Esta proposição ganha força ao se considerar que em outra comunidade periférica de Fortaleza (Conjunto Palmeiras), estudada por Monte (1992), observou-se comportamento similar. Nesta, ao mesmo tempo em que se afirmava que até os seis meses de idade o leite materno é o melhor e o mais seguro alimento, a sua utilização exclusiva era rara. Leite fraco, recusa do bebê e falta de paciência foram os argumentos apresentados pelos entrevistados.

Dentre a rede complexa de fatores que poderiam explicar tal ocorrência, a literatura tem destacado a baixa idade e escolaridade materna (Michaelsen et al., 1994a), a atuação do Setor Saúde, o poder da propaganda (Jones \& Green, 1993) e a grande influência de leigos (vizinhos, amigos, avós) (Pridham, 1990).

\section{Introdução dos alimentos de desmame}

Inicialmente, observou-se que mais de $50 \%$ das crianças em aleitamento parcial ou artificial (87), recebem água, chá, suco de frutas, leite em pó, farináceos, sopa, papa ou raspa de frutas como opção alimentar, sendo que a água, o chá, o leite em pó e os farináceos foram os alimentos com maior freqüência de consumo. Adicionalmente, a água, o chá, o leite em pó e os farináceos foram também os únicos itens da dieta com oferta iniciada antes do primeiro mês de vida. Os demais alimentos referidos foram introduzidos com maior freqüência a partir do terceiro mês (Figuras 1 e 2 ).

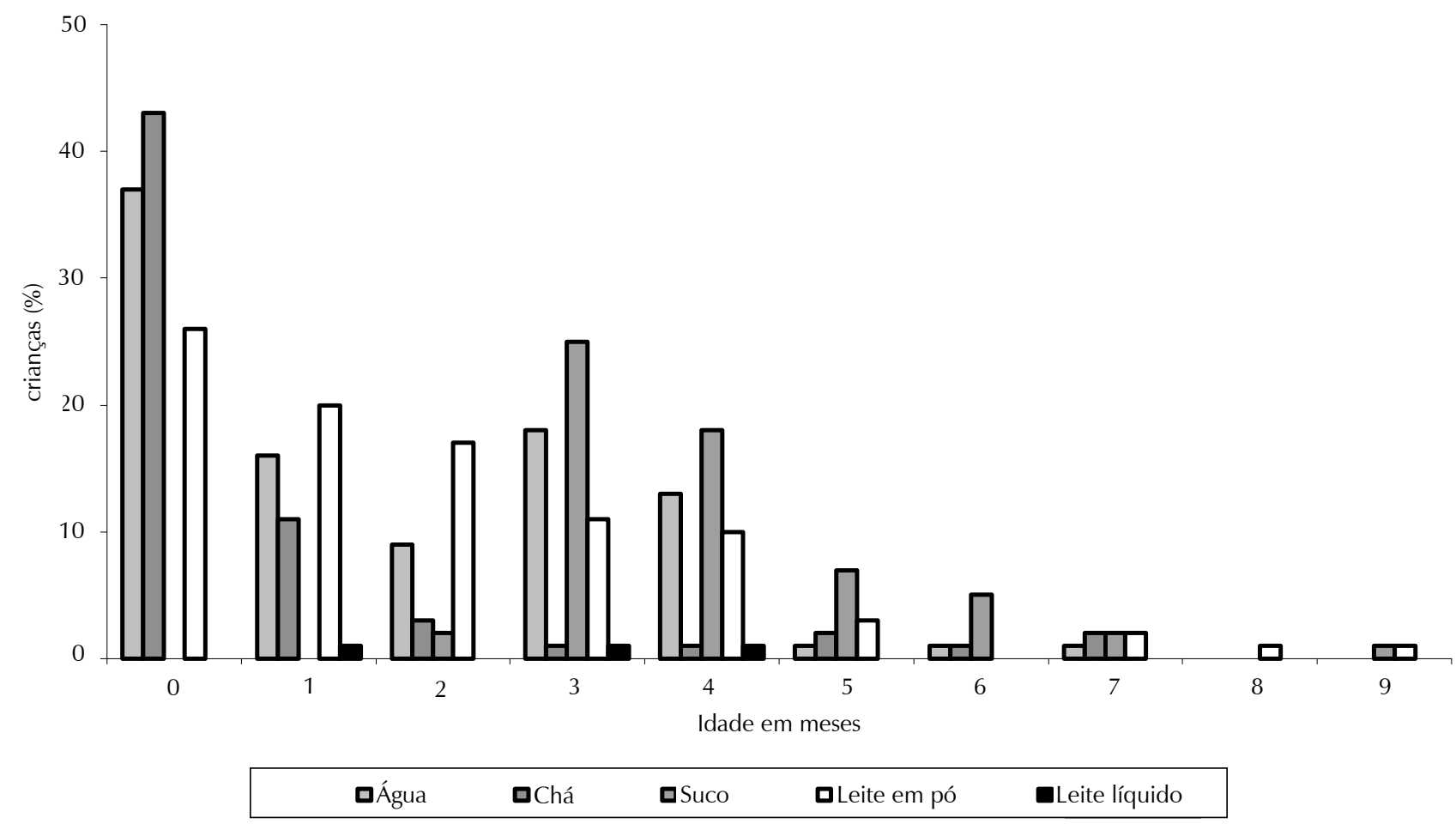

Figura 1. Freqüência de introdução dos alimentos líquidos em crianças < 1 ano. Álvaro Weine, Presidente Kennedy. Fortaleza, 1995. 


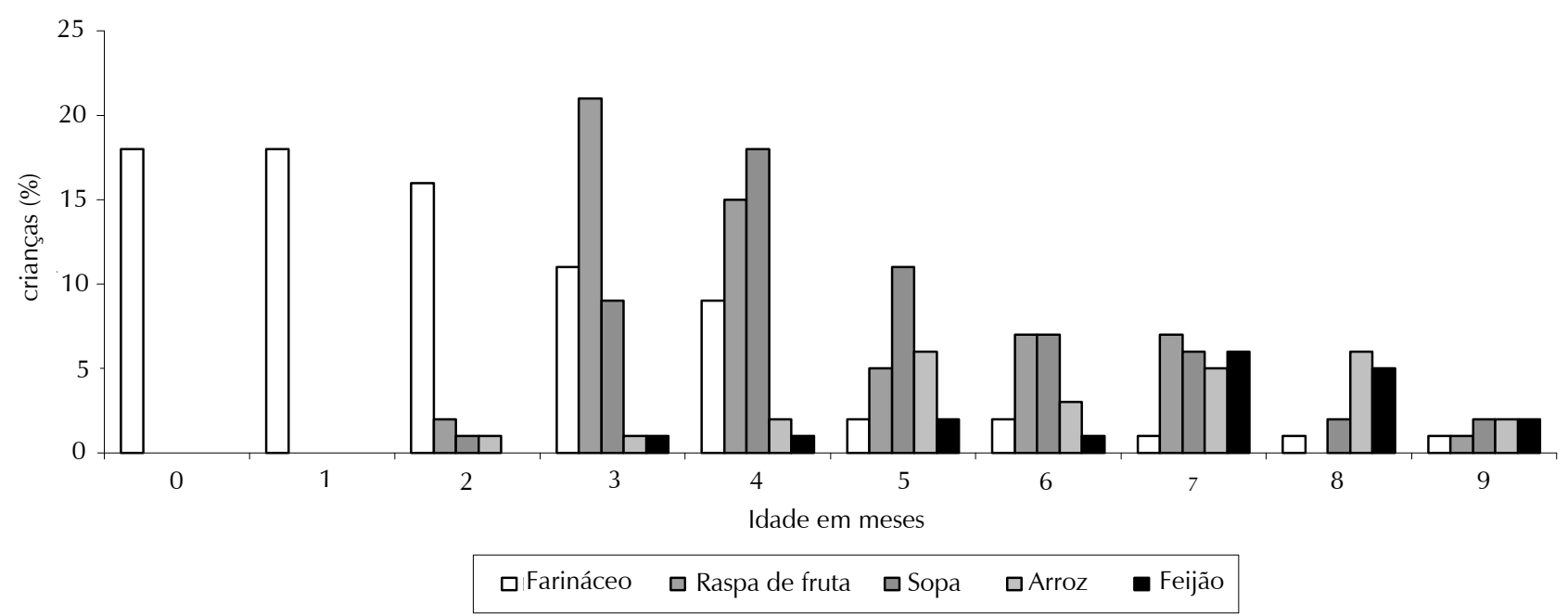

Figura 2. Freqüência de introdução dos alimentos sólidos em crianças < 1 ano. Álvaro Weine, Presidente Kennedy. Fortaleza, 1995.

O consumo diário de leite artificial foi observado em $89 \%$ das crianças já desmamadas, sendo que o seu consumo variou de 2 a 3 vezes por dia em $72 \%$ das crianças em aleitamento materno parcial (56\%).

Destaca-se que o leite de vaca líquido pasteurizado não constitui opção alimentar significativa no grupo estudado, pois somente três crianças o consomem. A aparente preferência da mãe pelo leite em pó chega a representar vantagem em relação ao líquido, devido à baixa disponibilidade de geladeira registrada nesta comunidade (40\%) (Soares, 1997).

Confrontando o perfil de introdução dos alimentos encontrado com as preconizações da literatura, verificou-se que além da oferta dos alimentos ser precoce, estes nem sempre são adequados a idade da criança ou representam riscos a sua sobrevivência. Independentemente do regime alimentar e mais precocemente no aleitamento materno parcial, observou-se que as crianças recebem alimentos fonte de carboidratos complexos (farináceos, arroz, feijão) antes do quarto mês de idade e ingerem alimentos semi-sólidos (sopa, papa ou raspa de frutas) antes dos 4-6 meses de idade, o que constitui uma prática anti-fisiológica (Organización Panamericana..., 1992).

A maioria das crianças também recebe durante os primeiros três meses de vida, água e chá, líquidos que, conjuntamente com a água de diluição do leite em pó, têm sido associados com significante aumento das infecções diarréicas (Kapil et al., 1994), problema que, por sinal, foi prevalente entre as crianças estudadas (Soares, 1997). Um estudo realizado em Pelotas, município da região mais desenvolvida do Brasil, demonstrou que as crianças que recebiam leite artificial (pó ou líquido) em adição ao leite materno, ou somente leite artificial, tinham risco 4,2 e 14,2 vezes maior de morte por diarréia, respectivamente, do que as crianças que não recebiam leite artificial (Victora,
1989). Cabe também considerar que a oferta precoce do leite de vaca favorece o mais rápido declínio da ingestão do leite materno e a maior possibilidade de desmame total durante o primeiro ano de vida (Drewett et al., 1993). Segundo a UNICEF, meio milhão de mortes poderiam ser evitadas anualmente, se o leite materno fosse uma prioridade na alimentação infantil (Hefti, 1993).

Paradoxalmente, enquanto os países desenvolvidos começam a experimentar o retorno à prática do aleitamento materno durante o primeiro ano de vida (Madhavapeddi \& Ramachandran, 1993), muitos países subdesenvolvidos têm mostrado declínio (Yunes et al., 1994), devido à rápida industrialização e elevação do status econômico (Singh et al., 1992).

\section{Alimentação básica}

As crianças estudadas têm acesso a uma considerável variedade de alimentos. Ao todo, listou-se o consumo de 48 produtos diferentes. Porém, ao se considerar básicos aqueles que são consumidos por pelo menos 50\% das crianças, verificou-se, na verdade, que a base da alimentação das crianças é composta por apenas 3 alimentos, além do leite materno: leite de vaca em pó integral, açúcar e farináceos (arrozina ${ }^{\circledR}$, maizena ${ }^{\circledR}$, mucilon ${ }^{\circledR}$, cremogema ${ }^{\circledR}$, farinha de trigo e goma), sendo que estes últimos foram principalmente consumidos na forma de mingau, preparação alimentar que já tem sido apontada como básica não só entre as crianças cearenses (Governo..., 1995), como também entre a maioria das crianças do mundo em desenvolvimento (Gopaldas, 1991; Ighogboja, 1992) .

A distribuição da freqüência de consumo por faixa etária demonstrou que esta base alimentar é exclusiva 
entre as crianças de zero a 3 meses de idade, enquanto que após o primeiro trimestre de vida outros alimentos são gradativamente introduzidos (banana, arroz). Maior variação no consumo de alimentos foi observada entre as crianças de 10-11 meses (banana, arroz, macarrão, iogurte, suco de laranja, batata inglesa, cenoura, chuchu, caldo de feijão), o que permite supor que esta faixa de idade é considerada pelas mães como o período mais apropriado para implementar a alimentação não láctea, com conseqüente abandono da oferta de leite materno (freqüência inferior a 50\%).

Ainda em conformidade com o que a literatura tem apontado para os países em desenvolvimento (Ighogboja, 1992; Chong-Ying et al., 1993; Ray et al., 1993) foi possível observar que alimentos, como vegetais folhosos e carnes (boi, peixe, frango) não figuram como padrão alimentar básico das crianças. Este fator, em adição à baixa variação e introdução tardia de frutas e hortaliças, sugerem implicações negativas no aporte de algumas vitaminas, minerais e fibras, o que poderá ser confirmado mais adiante.

Também merece destaque o consumo significativo de alimentos contra-indicados para os lactentes, como farináceos antes do quarto mês (Organización Panamericana..., 1992), açúcar refinado (Todd, 1992), leite de vaca integral (Redel \& Shulman, 1994) e iogurte (Carper, 1995). Registra-se que as proteínas do iogurte, alimento bastante consumido nos países mediterrâneos, podem provocar reações, causando diarréia, dificuldade de conciliar o sono, cólica e até alergias respiratórias (Carper, 1995).

Embora o consumo do leite em pó integral possa ser justificado pelo seu menor custo em relação ao modificado, no grupo estudado, segundo o relato das mães entrevistadas, o seu consumo está principalmente atrelado à crença de que esta é a melhor opção para o bebê e na preferência e/ou tolerância da criança. Mas, durante as entrevistas, percebeu-se que a preferência e a tolerância da criança recai muitas vezes na escolha do que a mãe considera como "melhor" para o seu filho. Além disto, ficou nítido em algumas entrevistas e subtendido em outras que o "padrão-ouro", em termos de leite artificial, é um tipo de leite em pó não modificado produzido pela Nestlé (Ninho ${ }^{\circledR}$ ), o qual é consumido por $51 \%$ das crianças que consomem leite em pó integral. O restante das crianças consomem outras marcas (Itambé ${ }^{\circledR}$ e Betânia $\left.{ }^{\circledR}\right)$ ). Vale ressaltar que, em comparação com o leite materno, o leite de vaca integral possui baixo teor de ferro, vitamina $\mathrm{E}$, ácido linoléico e contém excessiva quantidade de sódio, potássio e proteína (Bedinghaus \& Doughten, 1994).

Destaca-se que as mães que apresentaram justificativa de custo para respaldar o outro leite que estavam utilizando não a colocaram em relação ao leite modificado, mas sim em relação ao leite Ninho ${ }^{\circledR}$. No Conjunto Palmeiras, Monte (1992), encontrou-se mães preferindo utilizar um dos leites modificados mais caros do mercado, o Pelargon ${ }^{\circledR}$ (Nestlé). As mães alegaram ser melhor para o intestino da criança. Entre as crianças aqui estudadas, o consumo deste leite ficou em $6 \%$.

Quanto à alta freqüência de consumo do açúcar, embora a literatura apresente advertências sobre os seus malefícios (Todd, 1992), do ponto de vista quantitativo, o consumo médio per capita encontrado entre as crianças (35 g) não ultrapassou as recomendações de Araújo \& Guerra (1992) (37 g de 3-6 meses; 45 g de 6-9 meses), ficando em torno de $5 \%$ do Valor Energético Total (VET) das dietas consumidas. Brown \& Bégin (1993) referem que apesar da adição de óleo ou açúcar aumentar a densidade de energia dos alimentos infantis, pode ocorrer sobrediluição de nutrientes, com conseqüente comprometimento da adequação da dieta.

\section{Adequação de nutrientes e energia}

Comparando-se os dados contidos na Tabela 2, observa-se que as adequações da dieta artificial são superiores às da dieta mista, e que a consideração do consumo de leite materno favoreceu positivamente a adequação nutricional da dieta mista, como o esperado. Houve diferença estatisticamente significante entre as adequações nutricionais do aleitamento materno misto com e sem a estimativa do leite materno e aleitamento artificial $\left(\chi^{2}\right.$ de Friedman $\left.=12,6 ; p<0,05\right)$.

Há estudos indicando que a ingestão e a freqüência do aleitamento materno caem com a introdução de sólidos, ficando a ingestão energética 20\% abaixo das recomendações (National Research..., 1989), enquanto o mesmo não ocorre com as crianças alimentadas com fórmulas. Nestas, a ingestão energética permanece constante, ainda que a freqüência de oferta da fórmula decline. Ao contrário do que acontece com os amamentados ao seio, a energia dos sólidos é aditiva (Heinig et al., 1993). Pathak et al. (1993) citam que após os seis meses, quando outros alimentos, além do leite materno, representam maior proporção da ingestão total, o consumo de energia é mais baixo, mesmo quando a qualidade e quantidade dos alimentos oferecidos é similar. Michaelsen et al. (1994b) também registraram ingestão média de energia consideravelmente menor que as atuais recomendações em crianças em aleitamento materno, seja este, parcial ou exclusivo. Na Dinamarca (Taren \& Chen, 1993) e nos Estados Unidos (Heinig, 1993) foi observado, por sua vez, que crianças em aleitamento materno não apresentavam nenhuma injúria conseqüente da mais baixa ingestão de energia e menor ganho de peso, quando comparadas às alimentadas com fórmulas. Crianças em aleitamento materno podem ter diferente resposta fisiológica à ingestão de alimentos, a qual lhes permite consumir menos energia e ainda manter o crescimento (Taren \& Chen, 1993). 
Tabela 2. Distribuição das porcentagens de adequações médias de energia e nutrientes consumidos por crianças $<1$ ano, segundo as recomendações nutricionais (National Research..., 1989) e prática de aleitamento. Álvaro Weyne/Presidente Kennedy. Fortaleza, 1995.

\begin{tabular}{lccc}
\hline Energia e nutrientes & $\begin{array}{c}\text { Aleitamento misto sem adição do } \\
\text { leite materno* }\end{array}$ & Aleitamento misto & Aleitamento artificial \\
\hline Energia & 75 & 96 & 125 \\
Proteínas & 129 & 142 & 224 \\
Cálcio & 104 & 120 & 167 \\
Fósforo & 111 & 120 & 177 \\
Ferro & 28 & 29 & 25 \\
Vitamina C & 79 & 114 & 83 \\
Tiamina & 69 & 74 & 122 \\
Riboflavina & 181 & 200 & 314 \\
Niacina & 79 & 96 & 146 \\
Retinol & 69 & 111 & 155 \\
\hline
\end{tabular}

(*) O consumo de leite materno ficou estimado em $241 \mathrm{ml}$ no primeiro semestre e $193 \mathrm{ml}$ no segundo, em função do número médio de mamadas encontrado. $\left(\chi^{2}\right.$ de Fredman $\left.=12,6 ; p<0,05\right)$

Em adição a isto, não faltam dados na literatura destacando que o aleitamento materno é a melhor opção alimentar durante os primeiros meses de vida, não somente pelo aspecto nutricional, como por suas vantagens imunológicas, econômicas, emocionais e preventivas da morbi-mortalidade (Chandra et al., 1993; Taren \& Chen, 1993).

Portanto, além da estimativa de consumo do leite materno, há que se relevar na interpretação dos dados aqui apresentados, a possibilidade das crianças em regime alimentar parcial não estarem necessariamente em situação inferior às que estão em regime totalmente artificial, pois, pelo que se indica, o efeito dos componentes do leite materno na saúde infantil estão acima do método quantitativo. Vale ressaltar ainda que a análise nutricional de 600-800 $\mathrm{ml}$ de leite materno, que cobre, segundo Chandra et al. (1993) as necessidades do lactente até os 4-5 meses de idade, aponta déficit para a maioria dos nutrientes e energia. Somente a vitamina $\mathrm{C}$ e o retinol ficam adequados. Por outro lado, se as margens de segurança das Recommended Dietary Allowances (RDA) (National Research..., 1989) desfavorecem a adequação quantitativa do leite materno, as crianças em alimentação artificial podem, por conseguinte, estar em risco, não somente pelas deficiências observadas (vitamina C e ferro), mas por suas dietas ultrapassarem em mais que o dobro a adequação de alguns nutrientes, como é o caso das proteínas (224\%).

Entre 50 a 100\% das dietas mistas e artificiais, a riboflavina, o fósforo, o cálcio e a proteína foram os nutrientes que alcançaram as maiores porcentagens de adequação, estando todos disponíveis em boa quantidade no leite, ingrediente básico da alimentação das crianças. Porém, como a adequação média do ferro foi quase sempre a mais baixa, independentemente do padrão alimentar e faixa etária, chamou a atenção o fato de 3 $(18 \%)$ das crianças menores de seis meses em regime parcial serem as únicas a alcançarem as recomendações deste mineral ( $7 \%$ do total). Ao verificar a dieta consumida por estas mesmas crianças, encontrou-se em comum, o consumo concomitante de leite materno e leite em pó modificado, o primeiro numa quantidade média de $241 \mathrm{ml} /$ dia e o segundo de $120 \mathrm{~g} /$ dia. Destas crianças, somente uma consome outros alimentos (banana, água de coco, açúcar, suco de laranja).

A dieta de uma outra criança da mesma faixa etária, que também só consome leite materno e fórmula, não atingiu as recomendações de ferro porque a ingestão do leite artificial era inferior a média referida acima (62 g/dia). Desta forma, a utilização de fórmulas em quantidades adequadas parece ser fundamental na garantia do aporte de ferro de crianças em dietas mistas essencialmente lácteas.

É importante considerar que mesmo que o leite materno tivesse sido computado de forma mais precisa, os níveis de ferro, provavelmente, não atingiriam taxas desejáveis, pois o seu valor como fonte de ferro está ao nível da biodisponibilidade e não da quantidade. Por outro lado, o leite de vaca integral, que era amplamente consumido, além de possuir baixa quantidade de ferro, ainda está relacionado com perdas sangüíneas no intestino (Fuchs et al., 1993) e com a diminuição da biodisponibilidade do ferro da dieta (Cardoso \& Penteado, 1994).

\section{Distribuição percentual de carboidratos, lipídios e proteínas em relação ao VET}

A análise da distribuição dos macronutrientes em relação ao VET demonstrou que a maioria das dietas encontra-se dentro das faixas recomendadas pela literatura (Organização Mundial..., 1980; Anderson, 1988; Vannucchi et al., 1990). Cabe ressaltar, entretanto, que os limites percentuais atribuídos aos carboidratos (35-77,0\%) se enquadram no postulado de que estes têm função complementar (Vannucchi et al., 1990) aos valores 
recomendados para os lipídios (15-50,0\%) e proteínas (8-15,0\%). Contudo, nenhuma dieta apresentou percentual de carboidratos igual ou inferior a 35,0\%. Das que estavam abaixo de $50,0 \%$ de adequação $(14,0 \%)$, o percentual mínimo foi $43,0 \%$ e o máximo 49,8\% (média 46,0\%).

As dietas mistas foram superiores às artificiais no que tange aos intervalos recomendados dos macronutrientes e quanto a uma melhor distribuição média percentual destes. De acordo com os parâmetros utilizados, a dieta artificial tende para normo-protéica, hipolipídica e hiperglicídica, enquanto que a mista para normo-protéica, normoglicídica e normolipídica.

Destaca-se ainda que o teor médio de proteínas de alto valor biológico encontrado foi $90 \%$ (57 - 101), o que garante o aporte dos aminoácidos essenciais, em relação às calorias consumidas. $\mathrm{O}$ valor médio mínimo registrado foi $79 \%$ na faixa de $10-11$ meses do regime alimentar parcial.

Vale salientar que a correta proporção dos macronutrientes em relação às calorias totais não garante necessariamente a adequação dos micronutrientes. Alguns estudos sugerem que a falta de crescimento, ou subseqüente dificuldade de recuperá-lo, pode ocorrer mesmo quando a ingestão de energia e proteínas é adequada, aumentando a discussão da extensão da deficiência dos micronutrientes no prejuízo do crescimento linear (Allen, 1994).

\section{Relação cálcio : fósforo}

Dietas contendo baixa relação cálcio : fósforo (Ca : P) estão relacionadas com a ocorrência de tetania hipocalcêmica em recém-nascidos (Peckenpaugh \& Poleman, 1995) e parecem contribuir para o retardamento do crescimento linear, nos países em desenvolvimento (Waterlow, 1994).

Nos ossos jovens, a proporção de Ca para $P$ apresenta-se entre 1,7:1-2,14:1 g/g e nos ossos adultos em torno de 2,3:1 g/g (Prentice \& Bates, 1994).

O leite materno contém relativamente baixa quantidade de $\mathrm{P}$ e apresenta proporção Ca : P em torno de $2: 1 \mathrm{mg} / \mathrm{mg}$. No leite de vaca e maioria das fórmulas esta relação varia de 1,2 a 1,4:1. Desta forma, as crianças que consomem leite materno em regime parcial ou exclusivo ingerem menos P e mais Ca (Anderson, 1988; Prentice \& Bates, 1994). As RDA (National Research..., 1989) recomendam proporção 1,3: 1 (teor encontrado no leite de vaca integral) no primeiro semestre de vida e 1,2:1 no segundo. Segundo o Departamento de Saúde de Londres (Prentice \& Bates, 1994), a relação Ca : P, por si só, não parece ser fundamental, exceto nos países em desenvolvimento, onde a baixa proporção $\mathrm{Ca}$ : P está relacionada com alta ingestão de fitatos e oxalatos (compostos quelantes do cálcio), em decorrência do alto consumo de alimentos de origem vegetal. As RDA (National Research..., 1989) defendem parecer similar a partir do primeiro ano de vida.
Pôde ser observado que independentemente do padrão alimentar e faixa etária, a relação $\mathrm{Ca}$ : $\mathrm{P}$ da dieta consumida pelas crianças alcança em média as RDA (National Research......, 1989). No entanto, as crianças com idade acima de seis meses, em alimentação artificial, recebem dieta com baixa proporção destes minerais ( $1: 1)$. Relação mais elevada é observada entre as crianças com idade até seis meses $(1,4: 1)$ provavelmente, pelo predomínio da dieta láctea.

A despeito da maioria das dietas analisadas alcançarem, em média, as preconizações das RDA (National Research..., 1989), evidencia-se outro prejuízo da introdução precoce e/ou inadequada da alimentação artificial: o desbalanceamento da proporção Ca : P. Conforme sugere a literatura, o ideal seria que as crianças ingerissem no primeiro semestre de vida $\mathrm{Ca}: \mathrm{P}$ na proporção $2: 1$ (teor do leite materno) e que esta fosse caindo a 1,2:1 até o final do primeiro ano de vida, com a introdução da alimentação complementar.

\section{Teor de fibras}

A ingestão média de fibras foi inferior a 1,5 g/dia, sendo 0,4 g/dia entre os menores de seis meses e 1,4 g entre as crianças maiores. Considerando a proposição de Agostoni et al. (1995), que indica que a ingestão de fibras deve alcançar, gradativamente, $5 \mathrm{~g}$ /dia durante o segundo semestre de vida, a dieta consumida pelas crianças maiores de cinco meses poderia ser classificada como insuficiente em fibras. Ainda segundo estes mesmos autores, o excesso de proteína animal na dieta infantil, o qual chegou a ser evidenciado neste estudo, deveria ser corrigido com o aumento da ingestão de alimentos fontes de fibras (frutas, vegetais, legumes e cereais integrais), os quais também fornecem minerais e vitaminas (National Research..., 1989).

Apesar das indicações e contra-indicações das fibras na dieta infantil não terem sido ainda estabelecidas (Shneeman \& Tinker, 1995), a experiência clínica tem mostrado que a ingestão aumentada parece favorecer aquelas crianças até então alimentadas com dietas pobres em resíduos vegetais (Kelts \& Jones, 1988).

\section{CONCLUSÃO}

Há indicações de que a base alimentar das crianças estudadas constitui fator de risco para a saúde, a curto e a longo prazo. Desta forma, as instituições envolvidas com a assistência à saúde necessitam intensificar os esforços de promoção da prática alimentar infantil apropriada. Os prejuízos do aleitamento artificial e introdução inadequada dos alimentos de desmame devem ser mais amplamente discutidos e divulgados e as mães precisam conhecer os princípios fisiológicos e nutricionais que norteiam a alimentação da criança. 


\section{REFERÊNCIAS BIBLIOGRÁFICAS}

AGOSTONI, C., RIVA, E. GIOVANNINI, M. Dietary fiber in weaning foods of young children. Pediatrics, Evanston, v.9, n.11, Pt 2, p.1002-1005, 1995.

ALLEN, L.H. Nutritional influences on linear growth: a general review. European Journal of Clinical Nutrition, London, v.48, Supplement 1, p.75S-89S, 1994.

ANDERSON, L., DIBBLE, M.V., TURKKI, P.R., MITCHELL, H.S., RYNBERGEN, H.J. Nutrição. 17.ed. Tradução por Nadia Maria Frizzo Trugo. Rio de Janeiro : Guanabara, 1988. 737p. Tradução de: Nutrition in Health and Disease.

ARAÚJO, M.O.D., GUERRA, T.M.M. Alimentos per capita. Natal : Universitária, 1992. 181p.

BAVDEKAR, S.B., BAVDEKAR, M.S., KASLA, R.R., RAGHUNANDANA, K.J., JOSHI, S.Y., HATHI, G.S. Infant feeding practices in Bombay slums. Journal of the India Academy of Pediatrics, Calcutta, v.31, n.9, p.1083-1087, 1994.

BEDINGHAUS, J., DOUGHTEN, S. Childhood nutrition: from breastmilk to burgers. Primary Care; Clinics in Office Practice, Philadelphia, v.21, n.4, p.665-672, 1994.

BRASIL. Ministério da Saúde. Como ajudar as mães a amamentar. Brasília, 1994. 177p.

BROWN, K.H., BÉGIN, F. Malnutrition among wealings of developing countries: still a problem begging for solutions. Journal of Pediatric Gastroenterology and Nutrition, New York, v.17, n.2, p.132-138, 1993.

CARDOSO, M.A., PENTEADO, M.V.C. Intervenções nutricionais na anemia ferropriva. Cadernos de Saúde Pública, Rio de Janeiro, v.10, n.2, p.231-240, 1994.

CARPER, J. Alimentos, o melhor remédio para a boa saúde. Rio de Janeiro : Campus, 1995. 596p.

CHANDRA, R.K. Nutrition and immunity. Tropical and Geographical Medicine, Hague, v.40, n.3, p.46-51, 1988.

CHANDRA, R.K., BHAT, B., PURI, R.K. Why breast feed? Indian Pediatrics, New Delhi, v.30, n.6, p.841-851, 1993.

CHONG-YING, G. et al. Nutritional status of kadazan children in a rural district in Sabah, Malaysia. Southeast Asean J Trop Med Public Health, v.24, n.2, p.293, p.293-301, 1993.

COUTSOUDIS, A. et al. Determining appropriate nutritional interventions for South African children living in informal urban settlements. SAMJ, v.84, n.9, p.597-600, 1994.

DREWETT, R., AMATAYAKUL, K., WONGSAWASDII, L., MANGKLABRUKS, A., RUCKPAOPUNT, S., RUANGYUTTIKARN, C., BAUM, D., IMONG, S., JACKSON, D., WOOLRIDGE, M. Nursing frequency and the energy intake from breast milk and supplementary food in a rural Thai population: a longitudinal study. European Journal of Clinical Nutrition, London, v.47, p.880-891, 1993.

FUCHS, G.J. FARRIS, R.P., HUTCHINSON, S.N., WARRIER, R. Iron status and intake of older infants vs formula vs cow milk with cereal. American Journal of Clinical Nutrition, Bethesda. v.58, n.3, p.343-348, 1993.

GOPALDAS, T. Technologies to improve weaning foods in developing countries. Indian Pediatrics, New Delhi, v.28, n.3, p.217-221, 1991
GOVERNO DO ESTADO DO CEARÁ. A saúde das crianças cearenses. Fortaleza, 1990a. 73p.

GOVERNO DO ESTADO DO CEARÁ. II Pesquisa de saúde materno-infantil no Ceará - PESMIC II. Fortaleza, 1990b. $75 \mathrm{p}$.

GOVERNO DO ESTADO DO CEARÁ. III Pesquisa materno infantil do Ceará 1994 - PESMIC-3. Fortaleza, 1995. 61p.

HEFTI, R. Breastfeeding: then and now. The Canadian Nurse $L^{\prime}$ Infirmière Canadienne, v.89, n.9, p.38-39, 1993.

HEINIG, M.J., NOMMSEN, L.A., PEERSON, J.M., LONNERDAL, B., DEWEY, K.G. Intake and growth of breast-fed and formula-fed infants in relation to the timing of introduction of complementary foods: the DARLING study. Acta Paediatria, Oslo, v.82, n.12, p.999-1006, 1993.

HERVADA, A.R., NEWMAN, D.R. Weaning: historical perspectives, practical recommendations, and current controversies. Current Problems in Pediatrics, Chicago, v.22, n.5, p.223-240, 1992.

HUFFMAN, S.L., MARTIN, L. Child nutrition, birth spacing, and child mortality. Annals of the New Academy of Sciences, New York, v.709, p.236-248, 1994.

IGHOGBOJA, S. I. Some factors contributing to protein-energy malnutrition in the middle belt of Nigeria. East African Medical Journal, Nairobi, v.69, n.10, p.566-571, 1992.

INSTITUTO BRASILEIRO DE GEOGRAFIA E ESTATÍSTICA. Perfil estatístico de crianças e mães no Brasil: aspectos nutricionais 1974-75. Rio de Janeiro, 1982. 267p.

INSTITUTO BRASILEIRO DE GEOGRAFIA E ESTATÍSTICA. Censo demográfico 1991. Fortaleza, [199-]. (não paginado).

INSTITUTO BRASILEIRO DE GEOGRAFIA E ESTATÍSTICA. Crianças, adolescentes: indicadores sociais. Rio de Janeiro, 1992a. v.4: p.1-159.

INSTITUTO BRASILEIRO DE GEOGRAFIA E ESTATÍSTICA. Perfil estatístico de crianças e mães no Brasil: aspectos de saúde e nutrição, 1989. Rio de Janeiro, 1992b. 130p.

JONES, F., GREEN, M. Baby friendly care. The Canadian Nurse L'Infirmière Canadiense, v.89, n.9, p.1-7, 1993.

KAPIL, V., VERMA, D., NARULA, S., NAYAR, D., SACHDEV, H.P.S., SHAH, A.D., GNANASEKARAN, N. Breast-feeding practices in schedule caste communities in Haryana state. Indian Pediatrics, New Delhi, v.31, n.10, p.1227-1231, 1994.

KELTS, D.G.K., JONES, E.G. Manual de nutrição infantil. Tradução por Hildegard Thiemann Buckup. Rio de Janeiro : Guanabara, 1988. 312p. Tradução de: Manual of Pediatric Nutrition.

KUMAR, S., NATH, L.M., REDDAIAH. Supplementary feeding pattern in children living in a resetllement colony. Indian Pediatrics, New Delhi, v.29, n.2, p.219-222, 1992.

MADHAVAPEDDI, R., RAMACHANDRAN, P. Growth of urban breastfed infants from low socio-economic group. Journal of Tropical Pediatrics, London, v.39, p.328-331, Dec. 1993.

MARTINS, M.H.S. Valor nutritivo de alimentos definido por pesos médios, frações e medidas caseiras. Recife : UFPE, 1982. $109 \mathrm{p}$.

MICHAELSEN, K.F. LARSEN, P.S., THOMSEN, B.L., SAMUELSON, G. The Copenhagen cohort study on infant nutrition and growth: duration of breast feeding and influencing factors. Acta Paediatrica, Oslo, v.83, n.6, p.565-71, 1994a. 
MICHAELSEN, K.F., LARSEN, P.S., THOMSEN, B.L., SAMUELSON, G. The Copenhagen cohort study on infant nutrition and growth: breast-milk, human milk macronutrient content, and influencing factors. American Journal of Clinical Nutrition, Bethesda, v.59, n.3, p.600-611, 1994b.

MONTE, C.M.G. Improving weaning food hygiene practices in a slum area of Fortaleza, Northeast Brazil: a new approach. London, 1992. 293p. Thesis (Doctarate in Philosophy) - London School of Hygiene and Tropical Medicine, 1992.

NATIONAL RESEARCH COUNCIL (USA). Recommended Dietary Allowances. 10.ed. Washington DC : Nacional Academy Press, 1989. 285p.

ORGANIZAÇÃO MUNDIAL DE SAÚDE. Reunião conjunta OMS/ UNICEF sobre alimentação de lactentes e crianças na primeira infância. Brasília, 1980. 99p.

ORGANIZACIÓN PANAMERICANA DE LA SALUD. Alimentación infantil bases fisiológicas. Guatemala, 1992. 121p.

PATHAK, A., SHAH, N., TATARIA, A. Growth of exclusively breastfeed infants. Indian Pediatrics, New Delhi, v.30, n.11, p.1291-1300, 1993.

PECKENPAUGH, N.J. POLEMAN, C.M. Maternal and infant nutrition. In: PECKENPAUGH, N.J., POLEMAN, C.M. Nutrition essentials and diet therapy. 7.ed. Philadelphia: W.B. Saunders, 1995. Chapter 16: p.340-370.

PRADO, M.S., ASSIS, A.M.A, FREITAS, M.C.S., SILVA, R.C., VARJÃO, M.L. Padrão e seleção de alimentos complementares e sucedâneos do leite materno em comunidades rurais no semi-árido baiano. Revista de Nutrição da PUCCAMP, Campinas, v.8, n.1, p.47-64, 1995

PRENTICE, A., BATES, C.J. Adequacy of dietary mineral supply for human bone growth and mineralisation. European Journal of Clinical Nutrition, London, v.48, Supplement 1, p.161S-177S, 1994.

PRIDHAM, K.F. Feeding behaviour of 6 to 12 month-old infants: assessment and sources of parenteral information. Journal of Pediatrics, St. Louis, v.17, n.2, Part 2, p.174S-180S, 1990. Supplement.

RAY, B., BISWAS, R., CHOUDHURY, G., BISWAS, A.B. Infant feeding practices in a rural community of West Bengal. Indian Journal of Public Health, Calcutta, v.37, n.1, p.27, 1993.

REDEL, C.A., SHULMAN, R.J. Controversies in the composition if infant formulas. Pediatric Clinics of North America, Philadelphia, v.41, n.5, p.909-923, 1994.
SCHNEEMAN, B.O., TINKER, L.F. Dietary fiber. Pediatric Clinics of North America, Philadelphia, v.42, n.4, p.825-837, 1995.

SHRIMPTON, R. Ecologia da desnutrição na infância: análise da evidência das relações entre variáveis sócio-econômicas e estado nutricional. Brasília : UNICEF, 1986. 82p. (Série Instrumentos para Ação, 3).

SINGH, R., KUMAR, O.A., RANA, R.S. Breast-feeding and weaning practices among urban muslims of District Lucknow. Indian Pediatrics, New Delhi, v.29, n.2, p.217-219, 1992.

SOARES, N.T. Prática alimentar de crianças menores de um ano: um subsídio para implementação das ações em nutrição e saúde. Fortaleza, 1997. 177p. Dissertação (Mestrado em Saúde Pública) - Universidade Estadual do Ceará, 1997.

SZARFARC, S.C. et al. Estudo das condições de saúde das crianças do município de São Paulo, SP (Brasil), 1984/1985 x consumo alimentar. Revista da Saúde Pública, São Paulo, v.22, n.4, p.226-72, 1988.

TAREN, D., CHEN, J. A positive association between extended breast-feeding and nutritional status in rural Hubei Province, People's Republic of China. American Journal of Clinical Nutrition, Bethesda, v.58, n.6, p.862-867, 1993.

TODD, S. Weaning without sugar. Nursing Times, v.88, n.32, p.27-28, 1992.

UNDERWOOD, B.A., HOFVANDER, Y. Appropriate timing for complementary feeding of breast-fed infant: a review. Acta Paediatrica Scandinavica, Stockholm Supplement 294,.., 1982. 32p.

VANNUCCHI, H., MENEZES, E.W., CAMPANA, A.O., LAJOLO, F.M. Aplicação das recomendações nutricionais adaptadas à população brasileira. São Paulo : SBAN, 1990. 155p.

VICTORA, C. G. et al. Infant feeding and deaths due to diarrhea - a case-control study. American Journal of Epidemiology, v.129, n.5, p.1032-1041, 1989.

WATERLOW, J.C. Causes and mechanisms of linear growth retardation (stunting). European Journal of Clinical Nutrition, London, v.48, Supplement 1, p.1S-4S, 1994.

YUNES, J., CHELALA, C., BLAISTEIN, N. Children's health in the developing world: much remains to be done. World Health Forum, Geneva, v.15, n.1, p.73-76, 1994.

Recebido para publicação em 21 de outubro de 1998 e aceito em 18 de outubro de 1999. 